boceprevir have recently been approved in Europe in combination with pegINF and RBV for the treatment of patients with genotype $1 \mathrm{HCV}$ who have not been treated previously or when standard treatment has failed. They are serine protease inhibitors and belong to a new class of drugs: direct acting antivirals (DDAs).

Purpose To evaluate the pharmacoeconomic aspects of triple therapy with RBV, pegIFN and telaprevir or boceprevir, as reported in the literature.

Materials and Methods Cut-off guidelines have been established to quantify the suitability of new treatments based on the cost of treatment per quality-adjusted life year (OALY). The impact of using the new drugs was assessed on a hypothetical group of 14,000 patients infected with HCV (genotype 1). Unfortunately the price of the new drugs has not yet been negotiated in Italy; this represents a limit on the evaluation. The results are expressed in terms of Incremental cost-effectiveness ratios (ICERs).

Results The cost was estimated at $€ 31,000 /$ patient, $236.5 \mathrm{M} €$ over a period of 30 years. The ICER calculated to 20 years was $€ 29.485 /$ OALY while at 30 years was $€ 18.291 /$ OALY. Investment in these new molecules is favourable from a time horizon of 20 years.

Conclusions Boceprevir and telaprevir with standard treatment are cost effective considering the lifetime incidence of liver complications, quality-adjusted life years and the incremental costeffectiveness ratio. The cost effectiveness depends on the adherence to the treatment; it could be improved if the diagnostic and therapeutic pathways were optimised.

No conflict of interest.

\section{DGI-054 POST-PANDEMIC INFLUENZA A (H1N1) INFECTION IN CRITICALLY ILL PATIENTS PREVIOUSLY VACCINATED}

doi:10.1136/ejhpharm-2013-000276.320

${ }^{1} \mathrm{~L}$ Canadell Vilarrasa, ${ }^{2} \mathrm{AH}$ Rodriguez Oviedo, ${ }^{2} \mathrm{E}$ Diaz Santos. ${ }^{1}$ Hospital Universitari Joan XXIII de Tarragona, Pharmacy, Tarragona, Spain; ${ }^{2}$ Hospital Universitari Joan XXIII de Tarragona, ICU, Tarragona, Spain

Background The A H1N1 2009 virus caused a worldwide pandemic during 2009. Vaccination of high-risk individuals was one of the recommendations of the World Health Organization before the post-pandemic period. Since this period, influenza activity has again associated with A H1N1 virus in Spain.

1059 cases of severe flu were hospitalised during the postpandemic period in Spain and 41\% of them were admitted to the ICU. The status of influenza vaccination was determined in $92 \%$ of the ICU patients.

Purpose To compare differential characteristics in morbidity, mortality and clinical manifestations of vaccinated patients who were admitted to Spanish ICUs during the flu season 2010-11 versus unvaccinated patients.

Materials and Methods Prospective, observational and multicentre study performed in 148 ICUs. Data were recorded in the GTEI/ SEMICYUC registry. Adult patients with influenza A (H1N1) confirmed by rt-PCR were included in the analysis. Database records discriminated between having or not having been vaccinated.

Results 397 patients were admitted to Spanish ICUs during the post-pandemic period 2010/11 and supplied information about previous vaccination. A total of $22(5.8 \%)$ patients had previously been vaccinated

Vaccinated patients had a higher percentage of comorbidities compared to the other patients, $(95.5 \%$ vs. $74.1 \%$; $p=0.021)$. The mean number of comorbidities was also higher in vaccinated patients [1.91 (1.41) vs. 1.18 (0.99); $p=0.026]$.

Vaccinated patients showed higher rate of overall pneumonia but not bacterial coinfection. They received empiric antiviral treatment in a similar percentage and dosage, but they were treated for less time [6.9 (4.07) days vs. 8.99 (3.76) days; $p=0.003]$. There was
2 days of delay in the initiation of empiric antiviral treatment in vaccinated patients (7.64 vs. 5.59 days), although it was not statistically significant. Data also showed that a greater percentage of vaccinated patients were treated with zanamivir compared to the rest of the group ( $22.7 \%$ vs. $5.3 \% \mathrm{p}=0.008)$. Vaccinated patients did not differ from the rest of the group in time from onset of symptoms, days to hospital admission or time until diagnosis.

Conclusions Clinical presentation, management and antiviral treatment was different in patients who had been previously vaccinated against influenza $\mathrm{A}$ (H1N1) virus.

No conflict of interest.

\section{DGI-055 PROTEASE INHIBITORS: NEW DRUGS FOR TREATMENT OF CHRONIC HEPATIS C}

doi:10.1136/ejhpharm-2013-000276.321

M Pérez Abánades, C Martínez Nieto, E Alañón Plaza, A Aranguren Oyarzábal, E Deben Tiscar, E Ramírez Herráiz, T Gallego Aranda, A Ibañez Zurriaga, A Morell Baladrón. Hospital universitario la Princesa, Servicio de Farmacia, Madrid, Spain

Background The protease inhibitors boceprevir and telaprevir are indicated for treatment of chronic hepatitis $\mathrm{C}(\mathrm{CHC})$ genotype 1 in combination with peginterferon-alfa and ribavirin. These drugs increase efficacy and adverse effects.

Purpose To study the effectiveness and safety of boceprevir and telaprevir for treatment of CHC.

Materials and Methods Retrospective observational study including all patients who started treatment with telaprevir or boceprevir for treatment of CHC from January to September 2012.

Collected data: age, sex, type of patient (treatment-naive, recurrent or non-responder), liver fibrosis, HIV coinfection, viral loads at weeks $0,4,8,12,24$ to evaluate efficacy and adverse effects and supportive treatment to evaluate safety.

Results We included 51 patients, 35 (70\%) men and $15(30 \%)$ women, with a mean age of 51 years. 5 patients were co-infected with HIV (off-label use).

Abstract DGI-055 Table 1 Baseline characteristics

\begin{tabular}{lll}
\hline & Telaprevir & Boceprevir \\
\hline $\begin{array}{l}\text { Patients } \\
\text { Type of patient }\end{array}$ & $29(58 \%)$ & $21(42 \%)$ \\
treatment-naive & $5(17.24 \%)$ & $4(19.05 \%)$ \\
recurrent & $4(13.79 \%)$ & $10(47.62 \%)$ \\
non-responder & $20(68.97 \%)$ & $7(33.33 \%)$ \\
Liver fibrosis & & \\
$0-1$ & $6(20.69 \%)$ & $1(4.76 \%)$ \\
2 & $6(20.69 \%)$ & $2(9.52 \%)$ \\
$3-4$ & $17(58.62 \%)$ & $19(90.48 \%)$ \\
\hline
\end{tabular}

Abstract DGI-055 Table 2 Efficacy and safety

\begin{tabular}{lll}
\hline & Telaprevir & Boceprevir \\
\hline Negative viral loads at week & & \\
\hline 4 & $15 / 23(65.22 \%)$ & $7 / 15(46.67 \%)$ \\
8 & $18 / 21(85.71 \%)$ & $8 / 14(57.14 \%)$ \\
12 & $19 / 19(100 \%)$ & $4 / 5(80.00 \%)$ \\
24 & $8 / 8(100 \%)$ & $1 / 1(100 \%)$ \\
Anaemia & & \\
Reduced dose of ribavirin & $6(20.69 \%)$ & $6(28.57 \%)$ \\
Treatment with erythropoiesis-stimulating agent & $2(6.90 \%)$ & $1(4.76 \%)$ \\
Discontinued & $1(3.45 \%)$ & $1(4.76 \%)$ \\
Neutropenia & & \\
Reduction dose of peginterferon-alfa & $2(6.90 \%)$ & $4(19.05 \%)$ \\
Treatment with granulocyte colony-stimulating factor & $1(3.45 \%)$ & $4(19.05 \%)$ \\
(G-CSF) & & \\
Rash & & $0(0 \%)$ \\
Discontinued & $1(3.45 \%)$ & \\
\hline
\end{tabular}

